\title{
Time-dependent density-functional theory beyond the adiabatic approximation: Insights from a two-electron model system
}

\author{
C. A. Ullrich \\ Department of Physics and Astronomy, University of Missouri, Columbia, Missouri 65211
}

(Received 12 October 2006; accepted 14 November 2006; published online 21 December 2006)

\begin{abstract}
Most applications of time-dependent density-functional theory (TDDFT) use the adiabatic local-density approximation (ALDA) for the dynamical exchange-correlation potential $V_{\mathrm{xc}}(\mathbf{r}, t)$. An exact (i.e., nonadiabatic) extension of the ground-state LDA into the dynamical regime leads to a $V_{\mathrm{xc}}(\mathbf{r}, t)$ with a memory, which causes the electron dynamics to become dissipative. To illustrate and explain this nonadiabatic behavior, this paper studies the dynamics of two interacting electrons on a two-dimensional quantum strip of finite size, comparing TDDFT within and beyond the ALDA with numerical solutions of the two-electron time-dependent Schrödinger equation. It is shown explicitly how dissipation arises through multiple particle-hole excitations, and how the nonadiabatic extension of the ALDA fails for finite systems but becomes correct in the thermodynamic limit. (C) 2006 American Institute of Physics. [DOI: 10.1063/1.2406069]
\end{abstract}

\section{INTRODUCTION}

The essential ingredient of time-dependent densityfunctional theory (TDDFT), ${ }^{1,2}$ the exchange-correlation (xc) potential $V_{\mathrm{xc}}(\mathbf{r}, t)$, is frequently obtained using the adiabatic local-density approximation (ALDA):

$$
V_{\mathrm{xc}}^{\mathrm{ALDA}}(\mathbf{r}, t)=\left.\frac{d\left[\bar{n} e_{\mathrm{xc}}(\bar{n})\right]}{d \bar{n}}\right|_{\bar{n}=n(\mathbf{r}, t)},
$$

where $e_{\mathrm{xc}}(\bar{n})$ is the xc energy per particle of a homogeneous electron gas of density $\bar{n}$. The adiabatic approximation means that all functional dependence of $V_{\mathrm{xc}}(\mathbf{r}, t)$ on prior timedependent densities $n\left(\mathbf{r}^{\prime}, t^{\prime}\right), t^{\prime}<t$, is ignored. Neglecting the retardation implies frequency-independent and real $\mathrm{xc}$ kernels in linear response. This approach has been widely used in quantum chemistry for calculating molecular excitation energies. ${ }^{3}$

The adiabatic approximation is known to work well for excitation processes in many-body systems that have a direct counterpart in the Kohn-Sham system, such as atomic and molecular single-particle excitations. On the other hand, for more complicated processes such as double or chargetransfer excitations, the ALDA can fail dramatically. ${ }^{4,5}$ A recent study ${ }^{6}$ has shown that the ALDA can completely break down in dynamical processes where the electronic density rapidly undergoes large deformations.

Several recent papers have addressed the question how the LDA for ground-state calculations should be properly extended into the dynamical regime. ${ }^{6-12}$ Vignale and Kohn ${ }^{7}$ (VK) showed that a nonadiabatic local approximation for exchange and correlation requires the time-dependent current $\mathbf{j}(\mathbf{r}, t)$ as basic variable (C-TDDFT). The local C-TDDFT approximation of VK was later recast in the language of hydrodynamics, where xc effects beyond the ALDA appear as viscoelastic stresses in the electron liquid. ${ }^{9,10}$ An alternative nonadiabatic theory formulates TDDFT from the point of view of an observer in a comoving Lagrangian ref- erence frame (L-TDDFT). ${ }^{12}$ In Ref. 6 , the technical details of C-TDDFT and L-TDDFT (in the VK approximation and in the local deformation approximation, ${ }^{12}$ respectively) are critically examined and compared.

To date, most applications of TDDFT beyond the adiabatic approximation take place in the frequency-dependent linear-response regime. A major success of the VK formalism was the work by van Faassen et al. ${ }^{13,14}$ who calculated static axial polarizabilities in molecular chains, with much improvement over the ALDA.

The VK formalism has recently been applied to describe linear and nonlinear charge-density oscillations in quantum wells by solving the time-dependent Kohn-Sham (TDKS) equation. ${ }^{15}$ It was shown that the retardation caused by the memory of the xc potential has the striking consequence of introducing decoherence and energy relaxation, i.e., the oscillating density experiences a damping. The mechanism causing this behavior has been discussed by D'Agosta and Vignale. ${ }^{16}$ Technically, dissipation arises in VK theory from a velocity-dependent xc (vector) potential which breaks the time-reversal invariance of the TDKS Hamiltonian. As a result, a system tends to relax from a nonequilibrium initial state to an equilibrium final state with higher entropy. But where does the dissipated energy go?

Because the system is closed and isolated (there is no coupling to a thermal bath), the total energy should be conserved. According to Ref. 16, dissipation in C-TDDFT has to be understood in the sense that energy is redistributed between two subsystems with different sets of electronic degrees of freedom, coupled by Coulomb interactions. In the discussion of the quantum well examples of Refs. 15 and 16, the transfer of energy is said to occur from a collective motion along the confinement direction into low-lying lateral excitations of the two-dimensional electron gas in the quantum well plane. However, in C-TDDFT this transfer process is never directly observed, since the TDKS equations are solved only for the electron dynamics perpendicular to the 
quantum well plane. The damping of collective excitations in C-TDDFT as well as in the full many-body picture thus deserves further thought.

The purpose of the present paper is to give an explicit, pedagogical illustration of the road towards dissipation in collective electronic motion. We will consider a two-electron model system that is simple enough so that its dynamics can be treated numerically exactly via solution of the full timedependent Schrödinger equation and compare it with TDDFT within and beyond the ALDA. In particular, we will focus on charge-density oscillations along one direction of the system, and how the exact calculations show that the amplitude of these oscillations changes over time. This amplitude modulation comes from a superposition of transitions between the ground state and singly excited states and between singly and doubly excited states, including a coupling to the transverse degrees of freedom due to Coulomb interactions.

In ALDA, all effects involving multiple excitations are completely absent; in C-TDDFT in the local VK approximation, multiple excitations are implicitly included, but for finite systems their contribution is strongly exaggerated, producing an unphysical damping. Based on the insights of our simple two-electron system, we will discuss how the dissipative behavior emerges in the thermodynamic (largesystem) limit, and to what extent it is then correctly described by the VK formalism.

In Sec. II we give the technical details of our twoelectron model system and describe how the full Schrödinger equation and the TDKS equations with and without memory are solved. Section 3 gives our results and discusses the physical process of dissipation of collective charge-density oscillations. Conclusions are given in Sec. IV.

\section{MODEL SYSTEM}

Consider two electrons on a two-dimensional (2D) quantum strip of length $L$ and width $\Delta$, positioned in the $x-z$ plane. In the following, we will be mostly interested in situations where $L \gg \Delta$. The system has hard-wall boundary conditions at two ends of the strip, at $z=0$ and $z=\Delta$, and periodic boundary conditions along the $x$ direction. In other words, the electrons are living on a strictly 2D surface whose topology is equivalent to that of a cylindrical tube of length $\Delta$ and circumference $L$.

On this 2D quantum strip we first calculate the electronic ground state in the presence of a linear external potential which depends only on $z$ :

$$
V(z)=F z,
$$

where $F$ is a constant field strength. At the initial time $t=0$, this external potential is suddenly switched off, which triggers a charge-density oscillation along $z$ (see Fig. 1). The electronic density thus remains uniform along the $x$ direction for all times. The goal is to follow the time evolution of the system for many cycles of the charge-density oscillations, comparing the exact numerical solution of the two-electron Schrödinger equation with TDDFT solutions within and beyond ALDA. Atomic (Hartree) units are used throughout.
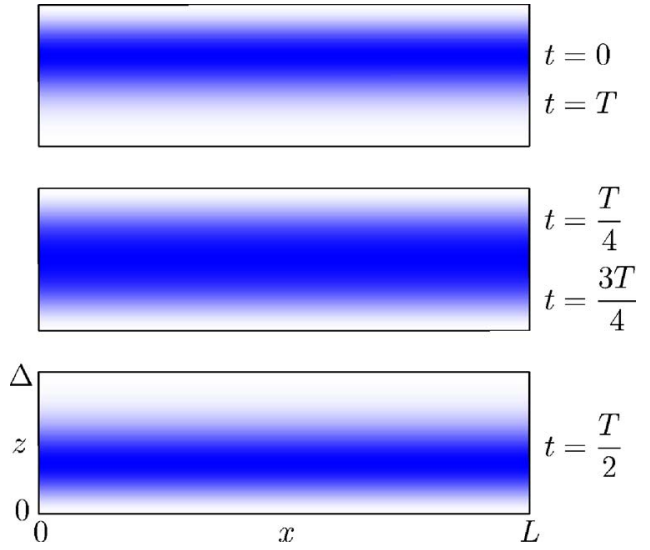

FIG. 1. (Color online) Schematic illustration of 1 cycle of a charge-density oscillation of a two-electron system on a $2 \mathrm{D}$ quantum strip of width $\Delta$ and length $L$. Darker areas represent regions of charge accumulation. Snapshots are shown at times as indicated, where $T$ is the duration of one cycle. The model assumes periodic boundaries along $x$ and hard-wall boundaries at $z=0$ and $z=\Delta$.

\section{A. Two-electron Schrödinger equation \\ 1. Ground state}

The static two-electron Schrödinger equation for our problem reads

$$
\begin{aligned}
0= & {\left[-\frac{\nabla_{1}^{2}}{2}-\frac{\nabla_{2}^{2}}{2}+V\left(z_{1}\right)+V\left(z_{2}\right)+\frac{1}{\left|\mathbf{r}_{1}-\mathbf{r}_{2}\right|}-E_{j}\right] } \\
& \times \Phi_{j}\left(\mathbf{r}_{1} s_{1}, \mathbf{r}_{2} s_{2}\right),
\end{aligned}
$$

where $\mathbf{r}_{1,2}=\left(x_{1,2}, z_{1,2}\right)$, and $s_{1,2}$ denotes the spin. We expand the two-electron eigenstates $\Phi_{j}$ in a basis of Slater determinants:

$$
\Phi_{j}\left(\mathbf{r}_{1} s_{1}, \mathbf{r}_{2} s_{2}\right)=\sum_{\substack{\nu_{1} \nu_{2} \\ \kappa_{1} \kappa_{2}}} C_{\nu_{1} \nu_{2} \kappa_{1} \kappa_{2}}^{j} \Psi_{\nu_{1} \nu_{2} \kappa_{1} \kappa_{2}}\left(\mathbf{r}_{1} s_{1}, \mathbf{r}_{2} s_{2}\right)
$$

where

$$
\begin{aligned}
\Psi_{\nu_{1} \nu_{2} \kappa_{1} \kappa_{2}}= & \frac{1}{\sqrt{2}}\left[\psi_{\nu_{1} \kappa_{1}}\left(x_{1}, z_{1}\right) \xi_{1}\left(s_{1}\right) \psi_{\nu_{2} \kappa_{2}}\left(x_{2}, z_{2}\right) \xi_{2}\left(s_{2}\right)\right. \\
& \left.-\psi_{\nu_{2} \kappa_{2}}\left(x_{1}, z_{1}\right) \xi_{2}\left(s_{1}\right) \psi_{\nu_{1} \kappa_{1}}\left(x_{2}, z_{2}\right) \xi_{1}\left(s_{2}\right)\right] .
\end{aligned}
$$

Here, $\xi$ are single-particle spinors, and for the spatial part we choose the noninteracting single-particle wave functions for constant external potential:

$$
\psi_{\nu \kappa}(x, z)=\sqrt{\frac{2}{L \Delta}} \exp \left(\frac{2 \pi i \kappa x}{L}\right) \sin \left(\frac{\pi \nu z}{\Delta}\right)
$$

with quantum numbers

$$
\begin{aligned}
& \kappa=0, \pm 1, \pm 2, \ldots, \\
& \nu=1,2,3, \ldots .
\end{aligned}
$$

In other words, we satisfy the given boundary conditions (see Fig. 1) by choosing plane-wave basis states along the strip and standing waves across the strip.

Inserting the basis expansion [Eq. (4)] into the Schrödinger equation [Eq. (3)] yields the following equation for the expansion coefficients: 


$$
\begin{aligned}
\sum_{\nu_{1} \nu_{2}}[ & \left(T_{\nu_{1} \kappa_{1}}+T_{\nu_{2} \kappa_{2}}\right) \delta_{\nu_{1} \mu_{1}} \delta_{\nu_{2} \mu_{2}} \delta_{\kappa_{1} \rho_{1}} \delta_{\kappa_{2} \rho_{2}} \\
& \left.+\left(V_{\mu_{1} \kappa_{2} \nu_{1}} \delta_{\nu_{2} \mu_{2}}+V_{\mu_{2} \nu_{2}} \delta_{\nu_{1} \mu_{1}}\right) \delta_{\kappa_{1} \rho_{1}} \delta_{\kappa_{2} \rho_{2}}+W_{\nu_{1} \nu_{2} \kappa_{1} \kappa_{2}}^{\mu_{1} \mu_{2} \rho_{1} \rho_{2}}\right] \\
& \times C_{\nu_{1} \nu_{2} \kappa_{1} \kappa_{2}}^{j}=E_{j} C_{\mu_{1} \mu_{2} \rho_{1} \rho_{2}}^{j} .
\end{aligned}
$$

Here and in the following, the indices $\kappa_{1,2}, \rho_{1,2}$ run over positive and negative integers including zero, whereas $\mu_{1,2}, \nu_{1,2}$ run over positive integers only [see Eqs. (7) and (8)]. The kinetic energy and external potential matrix elements are given by

$$
T_{\nu \kappa}=\frac{\pi^{2} \nu^{2}}{2 \Delta^{2}}+\frac{2 \pi^{2} \kappa^{2}}{L^{2}}
$$

and

$$
V_{\mu \nu}=\frac{2}{\Delta} \int_{0}^{\Delta} d z \sin (\tilde{\mu} z) \sin (\tilde{\nu} z) V(z),
$$

introducing the abbreviation $\tilde{\mu}=\pi \mu / \Delta$ and similar for $\widetilde{\nu}$. The matrix elements of the Coulomb interaction are

$$
\begin{aligned}
W_{\nu_{1} \nu_{2} \kappa_{1} \kappa_{2}}^{\mu_{1} \mu_{2} \rho_{1} \rho_{2}}= & \frac{4}{\Delta{ }^{2} L} \int_{0}^{\Delta} d z_{1} \int_{0}^{\Delta} d z_{2} \sin \left(\tilde{\mu}_{1} z_{1}\right) \sin \left(\tilde{\mu}_{2} z_{2}\right) \\
& \times \sin \left(\widetilde{\nu}_{1} z_{1}\right) \sin \left(\widetilde{\nu}_{2} z_{2}\right) \delta_{\kappa_{1}+\kappa_{2}, \rho_{1}+\rho_{2}} I_{\kappa_{2}-\rho_{2}}\left(z_{1}, z_{2}\right),
\end{aligned}
$$

where

$$
\begin{aligned}
I_{\kappa-\rho}\left(z_{1}, z_{2}\right) & =\int_{-\infty}^{\infty} d x \frac{\cos [2 \pi(\kappa-\rho) x / L]}{\sqrt{x^{2}+\left(z_{1}-z_{2}\right)^{2}}} \\
& =2 K_{0}\left[\frac{2 \pi}{L}|\kappa-\rho|\left|z_{1}-z_{2}\right|\right], \quad \kappa \neq \rho \\
& =-2 \log \left|z_{1}-z_{2}\right|, \quad \kappa=\rho .
\end{aligned}
$$

Here, $K_{0}$ is a complete Bessel function of the second kind in standard notation, and in the case $\kappa=\rho$ an additional divergent term is canceled by the positive background.

Solving Eq. (9) numerically one finds that a relatively small basis size including states with no more than $\kappa= \pm 10$ and $\nu=10$ [Eqs. (7) and (8)] is sufficient. The computational task is therefore quite manageable.

Furthermore, it turns out that, due to symmetry and momentum conservation, only those two-electron basis states $\Psi_{\nu_{1} \nu_{2} \kappa_{1} \kappa_{2}}$ contribute which have zero net current along the strip, i.e., only states with $\kappa_{1}=-\kappa_{2}$ are needed. This corresponds to two-electron states where one electron travels to the right and the other to the left.

\section{Time evolution}

Once Eq. (3) has been diagonalized, the next step is to determine the time evolution of the ground state $\Phi_{1}\left(\mathbf{r}_{1} s_{1}, \mathbf{r}_{2} s_{2}, t\right)$ after the linear external potential has been switched off. Rather than explicitly solving the time- dependent two-electron Schrödinger equation, this is most easily done by expanding $\Phi_{1}$ in the complete set of field-free eigenstates, defined as follows:

$$
\begin{aligned}
& {\left[-\frac{\nabla_{1}^{2}}{2}-\frac{\nabla_{2}^{2}}{2}+\frac{1}{\left|\mathbf{r}_{1}-\mathbf{r}_{2}\right|}-E_{j}^{f}\right] \Phi_{j}^{f}=0,} \\
& \Phi_{j}^{f}=\sum_{\substack{\nu_{1} \nu_{2} \\
\kappa_{1} \kappa_{2}}} C_{\nu_{1} \nu_{2} \kappa_{1} \kappa_{2}}^{j, f} \Psi_{\nu_{1} \nu_{2} \kappa_{1} \kappa_{2}} .
\end{aligned}
$$

Thus,

$$
\Phi_{1}(t)=\sum_{j} A_{j}(t) \Phi_{j}^{f},
$$

where

$$
A_{j}(t)=\exp \left[-i E_{j}^{f} t\right] \sum_{\substack{\nu_{1} \nu_{2} \\ \kappa_{1} \kappa_{2}}} C_{\nu_{1} \nu_{2} \kappa_{1} \kappa_{2}}^{j, f} C_{\nu_{1} \nu_{2} \kappa_{1} \kappa_{2}}^{1} .
$$

From this, we obtain the time-dependent density as follows:

$$
\begin{aligned}
n(z, t)= & \sum_{s_{1} s_{2}} \int d^{2} r_{2}\left|\Phi_{1}\left(\mathbf{r}_{1} s_{1}, \mathbf{r}_{2} s_{2}, t\right)\right|^{2} \\
= & \frac{2}{L \Delta} \sum_{\substack{\nu_{1} \nu_{2} \\
\kappa_{1} \kappa_{2}}} Q_{\mu_{1} \mu_{2} \nu_{1} \nu_{2}}(t)\left[\sin \left(\tilde{\mu}_{1} z\right) \sin \left(\widetilde{\nu}_{1} z\right) \delta_{\mu_{2}, \nu_{2}}\right. \\
& \left.+\sin \left(\tilde{\mu}_{2} z\right) \sin \left(\widetilde{\nu}_{2} z\right) \delta_{\mu_{1}, \nu_{1}}\right],
\end{aligned}
$$

where

$$
Q_{\mu_{1} \mu_{2} \nu_{1} \nu_{2}}(t)=\sum_{i j} A_{i}^{*}(t) A_{j}(t) \sum_{\kappa_{1} \kappa_{2}} C_{\nu_{1} \nu_{2} \kappa_{1} \kappa_{2}}^{i, f} C_{\nu_{1} \nu_{2} \kappa_{1} \kappa_{2}}^{j, f} .
$$

Finally, the time-dependent dipole moment is

$$
d(t)=\int_{0}^{\Delta} d z, z n(z, t) .
$$

\section{B. TDDFT}

\section{Ground state}

The two-electron problem described above can be solved, in principle exactly, using the TDKS formalism. We begin with the static Kohn-Sham (KS) equation:

$$
\left[-\frac{\nabla^{2}}{2}+V(z)+V_{H}(z)+V_{\mathrm{xc}}(z)-E_{n}\right] \phi_{n}(x, z)=0 .
$$

This equation separates in $x$ and $z$, and we make the ansatz

$$
\begin{aligned}
& \phi_{n}(x, z)=\frac{1}{\sqrt{L}} \exp \left(\frac{2 \pi i \kappa x}{L}\right) \varphi_{j}(z), \\
& E_{n}=\frac{2 \pi^{2} \kappa^{2}}{L^{2}}+\varepsilon_{j},
\end{aligned}
$$

where the index $\kappa$ is given by Eq. (7). The ground-state solution has $\kappa=0$, and we end up having to solve a onedimensional equation for $\varphi_{j}(z)$ and $\varepsilon_{j}$ : 


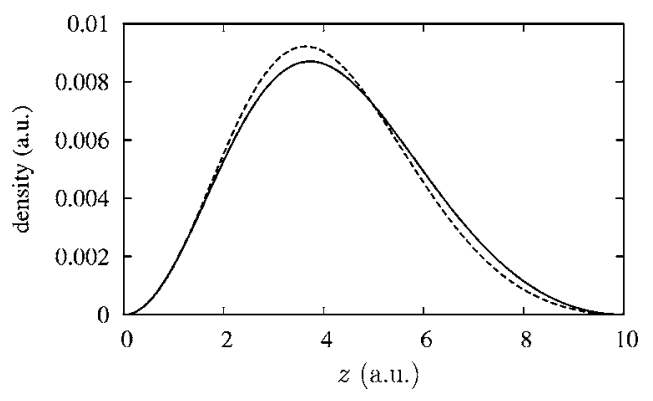

FIG. 2. Two-electron ground-state density $n(z)$ on a quantum strip of width $\Delta=10$ and length $L=50$, with field strength $F=0.02$. Full line: exact solution; dashed line: LDA.

$$
\left[-\frac{1}{2} \frac{d^{2}}{d z^{2}}+V(z)+V_{H}(z)+V_{\mathrm{xc}}(z)-\varepsilon_{j}\right] \varphi_{j}(z)=0 .
$$

To solve the single-particle $\mathrm{KS}$ equation, we expand in a standing-wave basis as follows:

$$
\varphi_{j}(z)=\sqrt{\frac{2}{\Delta}} \sum_{\nu=1}^{N} C_{\nu}^{j} \sin (\widetilde{\nu} z),
$$

where

$$
\sum_{\nu}\left[\frac{n^{2}}{2} \delta_{\mu \nu}+V_{\mu \nu}+V_{\mu \nu}^{H}+V_{\mu \nu}^{\mathrm{xc}}\right] C_{\nu}^{j}=\varepsilon_{j} C_{\mu}^{j} .
$$

From this, the ground-state density follows as

$$
n(z)=\frac{4}{L \Delta} \sum_{\mu \nu} C_{\mu}^{1 *} C_{\nu}^{1} \sin (\tilde{\mu} z) \sin (\widetilde{\nu} z) .
$$

The matrix elements for the external, Hartree, and xc potentials are calculated from Eq. (11). The Hartree potential is given by

$$
V_{H}(z)=-2 \int_{0}^{\Delta} d z^{\prime} n\left(z^{\prime}\right) \log \left|z-z^{\prime}\right|
$$

plus a diverging constant which is canceled by the positive background. For the xc potential we use the LDA within the parametrization of the 2D electron gas of Tanatar and Ceperley. ${ }^{17}$ For the (spin-unpolarized) systems under consideration, the more modern parametrization by Attaccalite et $a l .{ }^{18}$ gives almost identical results.

Figure 2 shows the two-electron ground-state density $n(z)$ on a $2 \mathrm{D}$ quantum strip of width $\Delta=10$ and length $L$ $=50$, in the presence of a linear external potential [Eq. (2)] with field strength $F=0.02$. For these system parameters, the 2D Wigner-Seitz radius $r_{s}=(\pi n)^{-1 / 2}$ has a value of $r_{s}=6$ at the maximum of the density distribution. The agreement between the exact and the LDA density is reasonably good and in fact becomes better for smaller quantum strips where the density is higher. In general, the LDA system is found to be a little more polarizable than the exact system.

\section{Time evolution}

Like in the case of the full two-electron Schrödinger equation, we set the charge-density oscillations in motion by suddenly switching off the external potential at the initial time $t=0$. The task is to solve the TDKS equation

$$
\left[-\frac{1}{2} \frac{d^{2}}{d z^{2}}+V_{H}(z, t)+V_{\mathrm{xc}}(z, t)-i \frac{\partial}{\partial t}\right] \varphi(z, t)=0,
$$

with initial condition $\varphi(z, 0)=\varphi_{1}(z)$. The time-dependent KS orbital $\varphi(z, t)$ is expanded similar to Eq. (26), and the timedependent expansion coefficients $C_{\mu}(t)$ are numerically determined using the Crank-Nicholson algorithm plus predictor-corrector scheme. ${ }^{2}$

In the following, we will consider $V_{\mathrm{xc}}(z, t)$ within and beyond the ALDA. In the VK approximation of C-TDDFT, one obtains an expression for a time-dependent xc potential with memory, called ALDA $+\mathrm{M}:^{6,15}$

$$
V_{\mathrm{xc}}(z, t)=V_{\mathrm{xc}}^{\mathrm{ALDA}}(z, t)+V_{\mathrm{xc}}^{M}(z, t),
$$

with the memory part

$$
V_{\mathrm{xc}}^{M}(z, t)=-\int_{0}^{z} \frac{d z^{\prime}}{n\left(z^{\prime}, t\right)} \nabla_{z^{\prime}} \sigma_{\mathrm{xc}, z z}\left(z^{\prime}, t\right) .
$$

The $z z$ component of the $\mathrm{xc}$ stress tensor is given by

$$
\sigma_{\mathrm{xc}, z z}\left(z^{\prime}, t\right)=\int_{0}^{t} Y\left(n\left(z^{\prime}, t\right), t-t^{\prime}\right) \nabla_{z^{\prime}} v_{z^{\prime}}\left(z^{\prime}, t\right) d t^{\prime} .
$$

Here, $\mathbf{v}(z, t)=\mathbf{j}(z, t) / n(z, t)$ is the time-dependent velocity field, where $\mathbf{j}(z, t)$ is the current density. In $2 \mathrm{D}$, the memory kernel $Y$ is given by

$$
Y\left(n, t-t^{\prime}\right)=\mu_{\mathrm{xc}}-\frac{n^{2}}{\pi} \int \frac{d \omega}{\omega} \Im f_{\mathrm{xc}}^{L}(\omega) \cos \left[\omega\left(t-t^{\prime}\right)\right],
$$

with the 2D xc shear modulus of the electron liquid ${ }^{19}$

$$
\mu_{\mathrm{xc}}=n^{2}\left(\Re f_{\mathrm{xc}}^{L}(0)-\left(n e_{\mathrm{xc}}\right)^{\prime \prime}\right)
$$

(the prime denotes a derivative with respect to $n$ ). In the following, we use the Holas-Singwi parametrization for the longitudinal frequency-dependent xc kernel of the 2D electron liquid: ${ }^{20}$

$$
\begin{aligned}
& \Im f_{\mathrm{xc}}^{L}(\omega)=\frac{A \omega}{B^{2}+\omega^{2}}, \\
& \Re f_{\mathrm{xc}}^{L}(\omega)=f_{\infty}+\frac{A B}{B^{2}+\omega^{2}} .
\end{aligned}
$$

The coefficients $A$ and $B$ are given by

$$
\begin{aligned}
& A=-\frac{11 \pi^{2}}{32}, \\
& B=\frac{A}{\left(n e_{\mathrm{xc}}\right)^{\prime \prime}-f_{\infty}},
\end{aligned}
$$

with

$$
f_{\infty}=\frac{1}{2 n}\left\{-\frac{5}{2} n^{2}\left(\frac{e_{\mathrm{xc}}}{n}\right)^{\prime}+12 n^{3 / 2}\left(\frac{e_{\mathrm{xc}}}{\sqrt{n}}\right)^{\prime}\right\} .
$$

It is easy to see that this simple parametrization for $f_{\mathrm{xc}}^{L}$ leads to zero shear modulus, $\mu_{\mathrm{xc}}=0$. A more sophisticated interpo- 


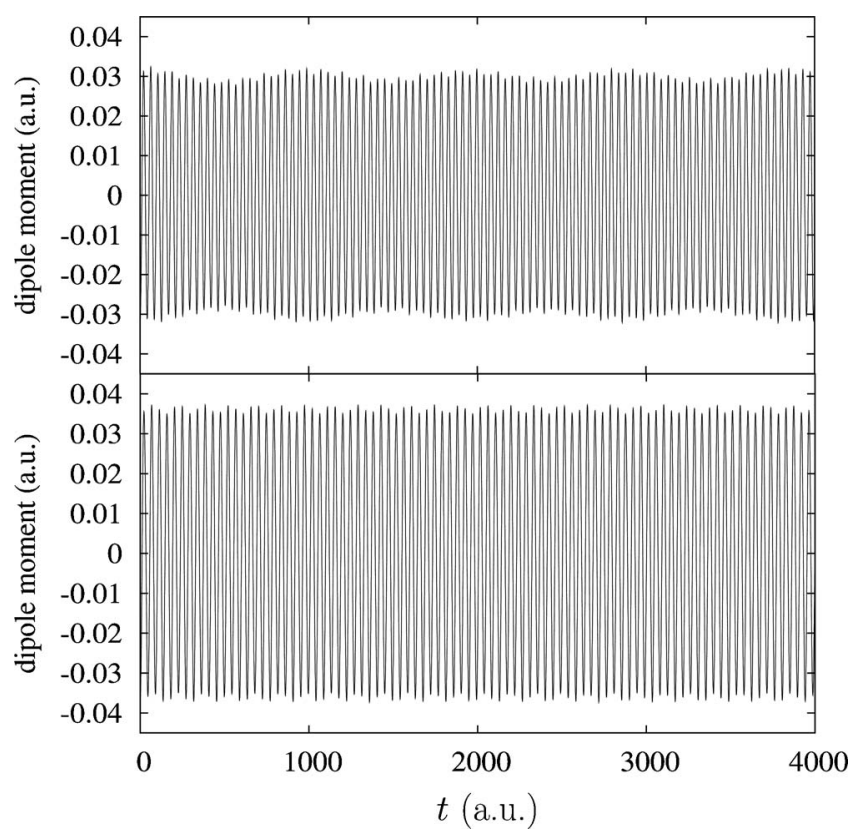

FIG. 3. Time-dependent dipole moment $d(t)$ associated with the chargedensity oscillations in a quantum strip of width $\Delta=10$, length $L=50$, and initial field strength $F=0.02$. Top: exact solution; bottom: ALDA.

lation formula, with finite $\mu_{\mathrm{xc}}$, has been derived by Qian and Vignale, ${ }^{19}$ but its input parameters are currently only available for a limited range of densities in the metallic regime. The shear modulus is known to be crucial for obtaining accurate static polarizabilities in polymers. ${ }^{13,14}$ However, here we are interested in a dynamical range of the order of the plasma frequency, and the three-dimensional (3D) study of Ref. 6 has shown that there is no qualitative, and not much quantitative, difference in that regime between $f_{\mathrm{xc}}^{L}$ parametrizations with zero and finite $\mu_{\mathrm{xc}}$ : both capture the mixed elastic/dissipative behavior of the electron liquid at finite frequencies. For our purposes, it is therefore sufficient, as well as practically more convenient, to work with the HolasSingwi formula [Eq. (36)].

An additional advantage of the Holas-Singwi $f_{\mathrm{xc}}^{L}$ is that it leads to a very simple expression for the memory kernel:

$$
Y\left(n, t-t^{\prime}\right)=-\frac{A n^{2}}{B} e^{-B\left(t-t^{\prime}\right)},
$$

i.e., the system experiences an exponential memory loss. This is similar to what was observed ${ }^{6,15}$ in 3D systems using the Gross-Kohn parametrization for $f_{\mathrm{xc}}^{L}{ }^{21}$ As a consequence, the numerical evaluation of the time integral in Eq. (33) can be simplified by introducing a cutoff in $t-t^{\prime}$, i.e., not the entire history of the system from $t=0$ onwards needs to be included.

\section{RESULTS AND DISCUSSION}

\section{A. Charge-density oscillations}

Figures 3 and 4 compare the time-dependent dipole moment $d(t)$ [Eq. (21)] for quantum strips of width $\Delta=10$ and lengths $L=50$ and $L=100$, respectively, calculated from the exact density [Eq. (19)] and from the ALDA density. The

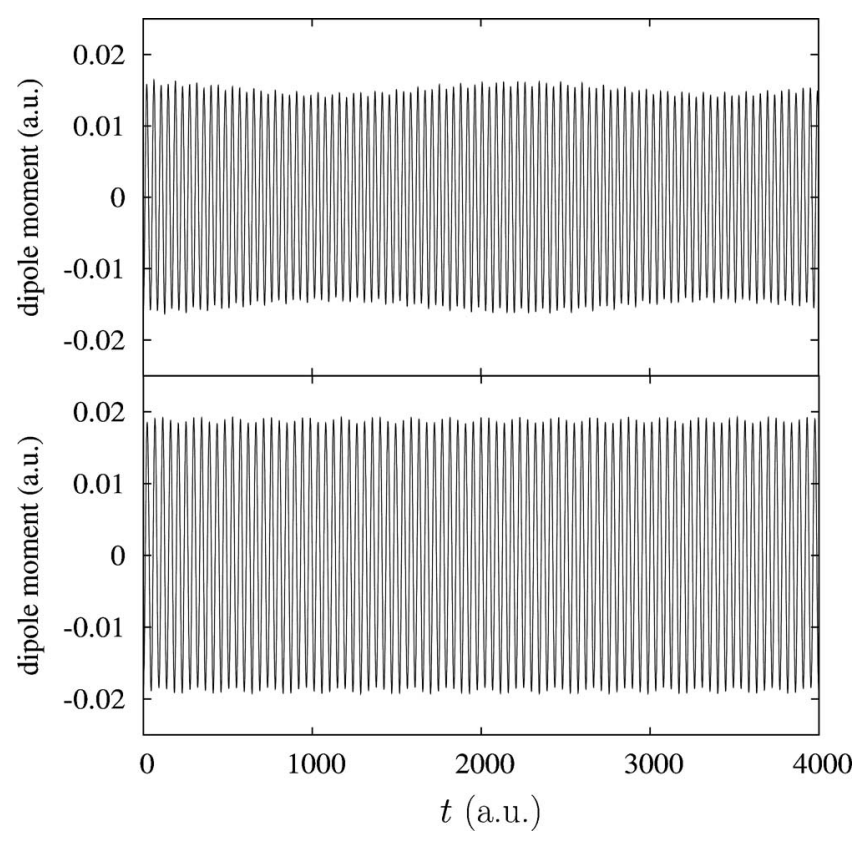

FIG. 4. Same as Fig. 3 but for a strip of length $L=100$.

initial state was prepared with an external potential [Eq. (2)] of field strength $F=0.02$, which was abruptly switched off at $t=0$.

At first sight, the ALDA charge-density oscillations seem to agree well with the exact ones, as far as the frequency and the average amplitude of $d(t)$ are concerned. On closer examination, however, we observe a beating pattern in the exact charge-density oscillations, which shows up as a lowfrequency modulation of the amplitude of $d(t)$. This effect is not reproduced by the ALDA, which produces a constant amplitude [we disregard here the small, rapid wiggles in the amplitudes of $d(t)$, which are a nonlinear effect caused by the relatively strong initial field, see Fig. 2]. In the following, we will focus on discussing the origin of these modulations, and on the related shortcomings of the ALDA and the consequences thereof. It will turn out that this effect provides a key to understanding the meaning of dissipation in TDKS theory.

In Sec. II A 2 we considered the time evolution of the exact two-electron state and explained how it can be obtained by expanding the time-dependent wave function in the complete set of field-free eigenstates, see Eq. (17). For the system parameters and field strengths under consideration, this expansion turns out to be dominated by just a few leading terms in the summation over $A_{j}(t) \Phi_{j}^{f}$. Looking at those few terms with the largest $\left|A_{j}(t)\right|^{2}$ will give us sufficient information to understand the electron dynamics leading to the beating pattern in $d(t)$.

Let us analyze in detail the case $\Delta=10, L=100$, and $F$ $=0.02$. We have solved the two-electron Schrödinger equation with 11 plane-wave and 8 standing-wave basis states, i.e., $|\kappa| \leqslant 5$ and $\nu \leqslant 8$ in Eqs. (7) and (8). Table I shows the six leading terms in the expansion [Eq. (17)] of $\Phi(t)$ in terms of field-free eigenstates, i.e., those terms with the largest $A_{j}^{2}$, and the associated energies $E_{j}$, where we define the field-free 
TABLE I. Leading terms in expansion (17) of the time-dependent twoelectron wave function $\Phi(t)$ in terms of field-free eigenstates (for a strip with $\Delta=10, L=100$, and $F=0.02)$. $\left(\nu_{1}, \nu_{2}\right)$ indicates the dominating singleparticle configurations (see Fig. 5).

\begin{tabular}{llcc}
\hline \hline$j$ & $\left(\nu_{1}, \nu_{2}\right)$ & $A_{j}^{2}$ & $E_{j}$ \\
\hline 1 & $(1,1)$ & 0.897406 & 0 \\
2 & $(1,2),(2,1)$ & 0.098766 & 0.148661 \\
3 & $(2,2)$ & 0.002698 & 0.294534 \\
4 & $(1,3),(3,1)$ & 0.001008 & 0.394043 \\
5 & $(2,3),(3,2)$ & 0.000051 & 0.542683 \\
6 & $(1,4),(4,1)$ & 0.000048 & 0.739263 \\
\hline \hline
\end{tabular}

ground-state energy to be $E_{1}=0$. The remaining terms in Eq. (17) have values of $A_{j}^{2}$ that are orders of magnitude smaller.

According to Eq. (4), each field-free eigenstate $\Phi_{j}^{f}$ is represented as a sum of single-particle Slater determinants. The leading $\Phi_{j}^{f}$ are dominated by configurations $\Psi_{\nu_{1} \nu_{2} \kappa_{1} \kappa_{2}}$ whose standing-wave quantum numbers $\left(\nu_{1}, \nu_{2}\right)$ are given in the second column of Table I and which can have a broad range of plane waves $\left(\kappa_{1}, \kappa_{2}\right)$. This is illustrated in detail in Fig. 5, which shows histograms of the coefficients $\left[C_{\nu_{1} \nu_{2} \kappa_{1} \kappa_{1}}^{j, f}\right]^{2}$ in the expansion $\Phi_{j}^{f}=\sum_{\kappa_{1} \kappa_{2}}^{\nu_{1} \nu_{2}} C_{\nu_{1} \nu_{2} \kappa_{1} \kappa_{2}}^{j, f} \Psi_{\nu_{1} \nu_{2} \kappa_{1} \kappa_{2}}$ of the first four leading field-free eigenstates. One can clearly see that there are dominating pairs of standing-wave quantum numbers $\left(\nu_{1}, \nu_{2}\right)$, which explains the assignment in the

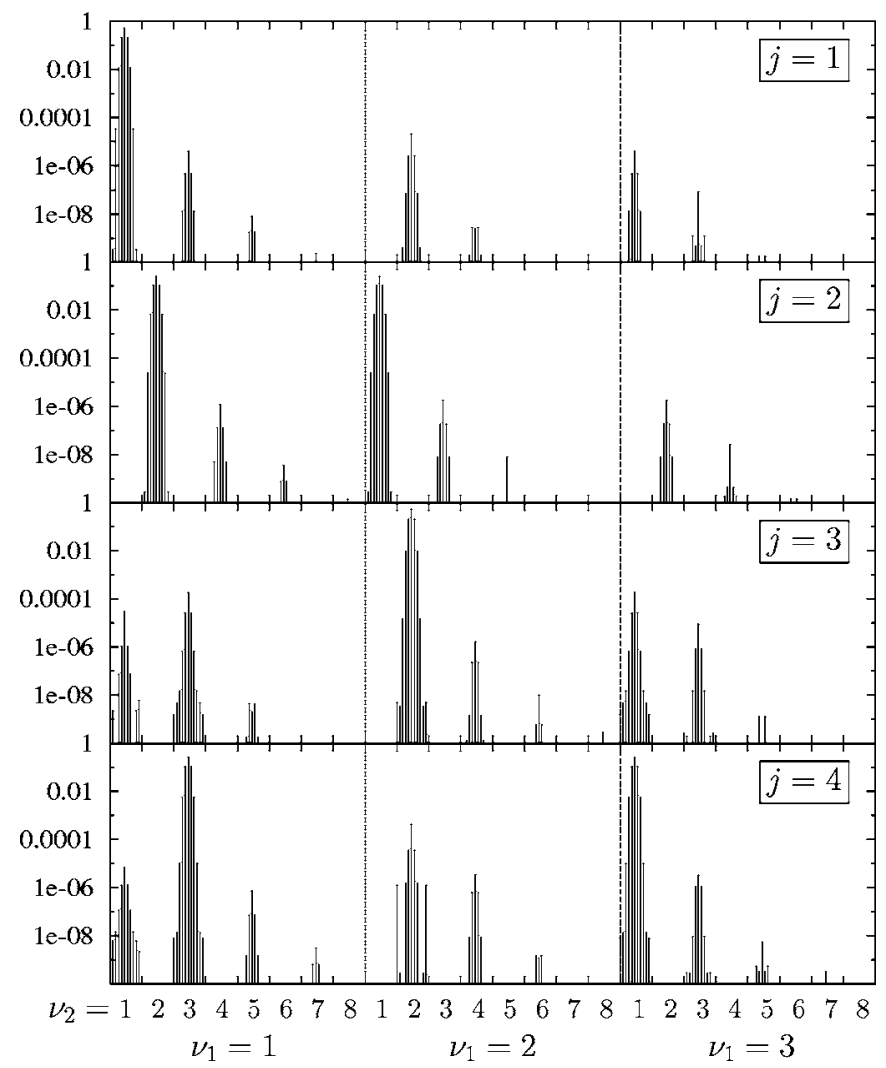

FIG. 5. Values of the coefficients $\left[C_{\nu_{1} \nu_{2} \kappa_{1} \kappa_{2}}^{j, f}\right]^{2}$ in the representation $\Phi_{j}^{f}$ $=\sum_{\kappa_{1} \kappa_{2}}^{\nu_{1} \nu_{2}} C_{\nu_{1} \nu_{2} \kappa_{1} \kappa_{2}}^{j, f} \Psi_{\nu_{1} \nu_{2} \kappa_{1} \kappa_{2}}$ of the first four leading field-free eigenstates (see Table I). Each configuration with standing waves $\left(\nu_{1}, \nu_{2}\right)$ along $z$ also has running waves along $x$ with $(\kappa,-\kappa)$, where $-5 \leqslant \kappa \leqslant 5$, shown here as histograms. The dominating configurations are for $\kappa=0$, but finite $\kappa$ are not negligible. second column of Table I. Each configuration with standing waves $\left(\nu_{1}, \nu_{2}\right)$ along $z$ is accompanied by left and right running waves $(\kappa,-\kappa)$ along $x$. The case $\kappa=0$ is dominant, but finite $\kappa$ are not negligible.

To understand the beating pattern in the dipole oscillations of Figs. 3 and 4, we now focus on the first three leading field-free eigenstates and their standing-wave quantum numbers $\left(\nu_{1}, \nu_{2}\right)$, and for the moment disregard the running waves along $x$. The beating pattern essentially arises from a superposition of two dipole oscillations associated with the transitions $(1,1) \rightarrow(1,2),(2,1)$ and $(1,2),(2,1) \rightarrow(2,2)$. The associated energy differences are $E_{2}-E_{1}=\omega_{21}$ $=0.148661$ and $E_{3}-E_{2}=\omega_{32}=0.145873$. The two oscillation frequencies $\omega_{21}$ and $\omega_{32}$ are very close, and their difference $\omega_{21}-\omega_{32}=0.002788$ is precisely the frequency of the amplitude modulation of $d(t)$. The resulting modulation period is $T_{\text {mod }}=2 \pi /\left(\omega_{21}-\omega_{32}\right)=2254$, which agrees extremely well with the data shown in Fig. 4. Similarly, for the case $L=50$ shown in Fig. 3 we find $T_{\text {mod }}=964$ (here, the difference $\omega_{21}-\omega_{32}$ is a bit bigger). The amplitude of the modulations of $d(t)$ depends on the field strength $F$ and remains small as long as $A_{1}^{2}, A_{2}^{2} \gg A_{3}^{2}$.

It is now easy to see why the ALDA misses the beating pattern in $d(t)$ : the reason is that it does not account for doubly excited configurations. The ALDA includes only single excitations, which are the only possible excitations of the KS system. Thus, transitions involving the $\left(\nu_{1}, \nu_{2}\right)$ $=(2,2)$ configuration, which are crucial to explaining the modulation of $d(t)$, cannot occur, and therefore no superposition effect takes place.

In addition to the standing-wave double excitations, the contribution of doubly excited running-wave states $(\kappa,-\kappa)$ along $x$ are also important. Again, the ALDA only includes the case $\kappa=0$ (single excitations along $x$ are not possible due to momentum conservation). On the other hand, ignoring the states $(\kappa,-\kappa)$ with finite $\kappa$ in the expansion of the full twoelectron wave function would lead to substantially different energies $E_{j}$, and the low-frequency beating pattern of $d(t)$ would be destroyed.

The exact xc potential (which we will construct in the next subsection) has to compensate for the absence of multiple excitations in the TDKS wave function, and it does so through a nonadiabatic contribution. This is known from linear-response theory, ${ }^{4}$ where the xc kernel must have a frequency dependence to describe double excitations.

\section{B. Exact xc potential and time-dependent energy 1. Construction of the exact time-dependent xc potential}

If the density $n(\mathbf{r}, t)$ of a system of two electrons in a singlet state is given, it is a straightforward affair to construct that xc potential $V_{\mathrm{xc}}(\mathbf{r}, t)$ which, when employed in a TDKS equation, reproduces this density. ${ }^{22}$ The doubly occupied TDKS orbital can be written as 


$$
\varphi(\mathbf{r}, t)=\sqrt{\frac{n(\mathbf{r}, t)}{2}} e^{i \alpha(\mathbf{r}, t)},
$$

where the phase $\alpha$ is a real function and related to the current density as follows:

$$
\nabla \alpha(\mathbf{r}, t)=\mathbf{j}(\mathbf{r}, t) / n(\mathbf{r}, t) .
$$

Inserting ansatz (42) into the TDKS equation, one obtains

$$
V_{\mathrm{xc}}(\mathbf{r}, t)=V_{\mathrm{xc}}^{\mathrm{stat}}(\mathbf{r}, t)+V_{\mathrm{xc}}^{\mathrm{dyn}}(\mathbf{r}, t) \text {. }
$$

The first term,

$$
\begin{aligned}
V_{\mathrm{xc}}^{\mathrm{stat}}(\mathbf{r}, t)= & \frac{1}{4} \nabla^{2} \ln n(\mathbf{r}, t)+\frac{1}{8}|\nabla \ln n(\mathbf{r}, t)|^{2}-V(\mathbf{r}, t) \\
& -V_{H}(\mathbf{r}, t),
\end{aligned}
$$

is identical to the expression for constructing the static $\mathrm{xc}$ potential from a given static two-electron density, ${ }^{23}$ except that all quantities are now taken as time dependent. The second term,

$$
V_{\mathrm{xc}}^{\mathrm{dyn}}(\mathbf{r}, t)=-\dot{\alpha}(\mathbf{r}, t)-\frac{1}{2}|\nabla \alpha(\mathbf{r}, t)|^{2},
$$

has no static counterpart and is therefore a truly dynamical contribution.

\section{Exact energy of the TDKS system}

Let us now consider the time dependence of the total energy $E(t)$ of our two-electron system. Since we are dealing with free charge-density oscillations of a finite 2D quantum strip, i.e., there is no time-dependent external force, the total energy of the full many-body system must obviously be constant. This is easy to see for the exact time-dependent twoelectron wave function $\Phi(t)$ : according to Eqs. (17) and (18), we simply have $E(t)=\sum_{j} E_{j}$ for all times.

As long as the external potential $V$ remains static, it is straightforward to express the constant total energy as a time-dependent density functional. ${ }^{24}$ To be as general as possible, we work within C-TDDFT, where the TDKS Hamiltonian is as follows: $:^{25,26}$

$$
\hat{h}(t)=\frac{1}{2}\left(\frac{\nabla}{i}+\frac{1}{c} \mathbf{A}_{\mathrm{xc}}(\mathbf{r}, t)\right)^{2}+V(\mathbf{r})+V_{H}(\mathbf{r}, t)+V_{\mathrm{xc}}(\mathbf{r}, t),
$$

where $\mathbf{A}_{\mathrm{xc}}(\mathbf{r}, t)$ is the xc vector potential. In particular, this applies for situations where the system evolves from an initial state that is not the ground state. Formally, the exact $V_{\mathrm{xc}}(\mathbf{r}, t)$ and $\mathbf{A}_{\mathrm{xc}}(\mathbf{r}, t)$ are therefore functionals of not only the time-dependent density but also of the initial many-body and KS wave functions. ${ }^{27}$

Generalizing the work of Hessler et al. ${ }^{28,29}$ to include the presence of $\mathbf{A}_{\mathrm{xc}}$, one can write for a two-electron system

$$
\begin{aligned}
E= & 2 \int d \mathbf{r} \varphi^{*}(\mathbf{r}, t)\left[\frac{1}{2}\left(\frac{\nabla}{i}+\frac{1}{c} \mathbf{A}_{\mathrm{xc}}(\mathbf{r}, t)\right)^{2}+V(\mathbf{r})\right] \varphi(\mathbf{r}, t) \\
& +E_{H}[n(t)]+E_{\mathrm{xc}}(t) .
\end{aligned}
$$

$E_{H}[n]$ is the Hartree energy functional, and the timedependent xc energy is defined by Eq. (48). One finds, using Eqs. (47) and (48),

$$
\dot{E}_{\mathrm{xc}}(t)=\int d \mathbf{r} \dot{n}(\mathbf{r}, t) V_{\mathrm{xc}}(\mathbf{r}, t)-\frac{1}{c} \int d \mathbf{r j}(\mathbf{r}, t) \cdot \dot{\mathbf{A}}_{\mathrm{xc}}(\mathbf{r}, t) .
$$

In Refs. 28 and 29 the main purpose of introducing timedependent energy components was to derive exact conditions and constraints for the scalar xc potential in TDDFT. It was found that the time-dependent correlation energy, obtained from Eq. (49), can sometimes become positive.

\section{The adiabatic energy}

To guarantee that the total $E$, Eq. (48), is constant, $E_{\mathrm{xc}}(t)$ has to be an extremely complicated functional whose dependence on density and current is nonlocal in space and time, and which also depends implicitly on the initial KS and many-body states.

A simple approximation to the time-dependent xc energy functional is obtained by taking the ground-state xc energy functional $E_{\mathrm{xc}}^{\mathrm{gs}}[n]$ that was used in the calculation of the initial state for the TDKS time propagation and evaluating it with the time-dependent density. Making this approximation in Eq. (48) defines the adiabatic energy $E_{a}(t)$ which was first introduced in Ref. 15 and more thoroughly analyzed by D'Agosta and Vignale. ${ }^{16}$ For a two-electron KS system with a doubly occupied single-particle orbital, we thus have

$$
\begin{aligned}
E_{a}(t)= & 2 \int d \mathbf{r} \varphi^{*}(\mathbf{r}, t)\left[\frac{1}{2}\left(\frac{\nabla}{i}+\frac{1}{c} \mathbf{A}_{\mathrm{xc}}(\mathbf{r}, t)\right)^{2}\right. \\
& +V(\mathbf{r})] \varphi(\mathbf{r}, t)+E_{H}[n(t)]+E_{\mathrm{xc}}^{\mathrm{gs}}[n(t)] .
\end{aligned}
$$

Clearly, $E_{a}(t)$ is not equal to the true total energy (which should be constant), but, just as the exact $E$ from Eq. (48), it reduces to the ground-state energy in the static limit. One finds that the rate of change of the adiabatic energy is

$$
\dot{E}_{a}(t)=\frac{1}{c} \int d \mathbf{r j}(\mathbf{r}, t) \cdot \dot{\mathbf{A}}_{\mathrm{xc}}(\mathbf{r}, t) .
$$

D'Agosta and Vignale ${ }^{16}$ proved that $E_{a}(t)$ decreases monotonically in the absence of an external driving field when the VK expression is used for $\mathbf{A}_{\mathrm{xc}}$, which indicates that the system is irreversibly driven to equilibrium.

In the case of our 2D quantum strip, the density is spatially inhomogeneous along the $z$ direction only, which means that we can replace the xc vector potential by the dynamical scalar potential using the relation

$$
\frac{1}{c} \dot{\mathbf{A}}_{\mathrm{xc}}(\mathbf{r}, t)=-\nabla V_{\mathrm{xc}}^{\mathrm{dyn}}(\mathbf{r}, t) .
$$

Furthermore, the $z$ components of the physical current $j_{z}(z, t)$ and the KS current $j_{\mathrm{KS}, z}(z, t)=2 \Im\left(\varphi^{*} d \varphi / d z\right)$ of this particular two-electron system become identical. Therefore, we obtain

$$
\dot{E}_{a}(t)=-L \int_{0}^{\Delta} d z j_{z}(z, t) \frac{d}{d z} V_{\mathrm{xc}}^{\mathrm{dyn}}(z, t) .
$$

This shows that the rate of change of the adiabatic energy is determined by the work done by the forces associated with 


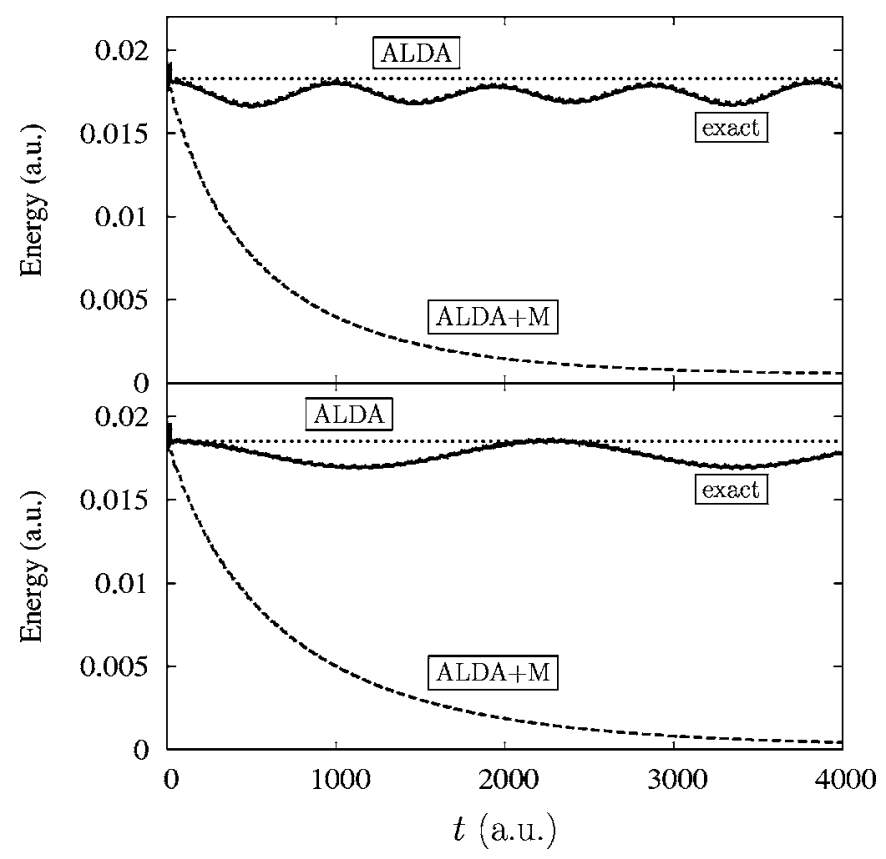

FIG. 6. Full lines: cycle-averaged adiabatic energy $\bar{E}_{a}(t)$ for the exact solutions of the charge-density oscillations on the 2D quantum strip with $L=50$ (top) and $L=100$ (bottom). Short and long dashed lines: $E_{a}(t)$ calculated with ALDA and ALDA+M. For clarity, all energies are shifted so that they initially coincide.

the dynamical xc potential. Thus, the adiabatic energy is a useful tool to analyze dissipation in TDDFT.

In the case where we start from the exact time-dependent density and ask what the associated exact adiabatic energy is, a direct evaluation of expression (50) is not possible, since one does not know the form of the exact xc energy functional. Fortunately, the adiabatic energy can easily be obtained from Eq. (53) through a simple time integration: $E_{a}(t)=\int_{0}^{t} \dot{E}_{a}\left(t^{\prime}\right) d t^{\prime}$. This is a very convenient way of determining the exact adiabatic energy (up to an irrelevant constant) from the exact density.

We have calculated $E_{a}(t)$ for the exact solutions of the charge-density oscillations for the 2D quantum strips with $L=50$ and $L=100$; see top parts of Figs. 3 and 4. As expected, the exact adiabatic energy is not constant but rather rapidly fluctuates with time. For the sake of clarity, and since these rapid fluctuations are not what we are primarily interested in, we define a cycle-averaged adiabatic energy $\bar{E}_{a}(t)$, which is obtained by averaging the adiabatic energy at each time $t$ over a time window of one period of the chargedensity oscillation (duration $\sim 40$ a.u.).

Figure 6 shows the adiabatic energy for the two quantum strips, calculated with different methods [both the ALDA and $\mathrm{ALDA}+\mathrm{M}$ energies have been directly determined from Eq. (50); the ALDA+M results will be discussed in the next subsection]. One clearly sees slow oscillations of the exact $\bar{E}_{a}(t)$ with the same period as the amplitude modulations of $d(t)$ (see Figs. 3 and 4). By contrast, $E_{a}(t)$ in ALDA is constant as expected, since the ALDA dipole amplitudes are not modulated.

These results provide some interesting insights into TDDFT. It follows from the Runge-Gross theorem ${ }^{1}$ that there

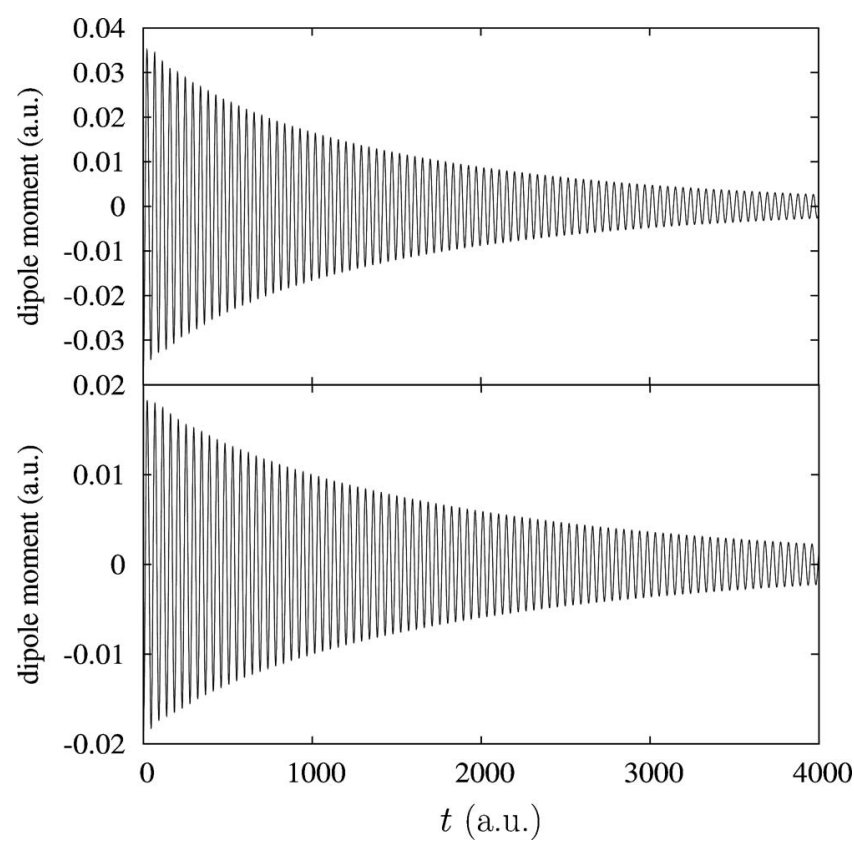

FIG. 7. Time-dependent dipole moment calculated with ALDA+M for the same chargedensity oscillations treated in Figs. 3 and 4. Upper panel: $L=50$; lower panel: $L=100$.

exists a unique TDKS system which reproduces any (reasonably well-behaved) time-dependent density $n(\mathbf{r}, t)$ of a manybody system. In particular, if the exact functional for the xc potential $V_{\mathrm{xc}}(\mathbf{r}, t)$ is used, the TDKS system gives the exact time-dependent density. However, the adiabatic energy (as defined above) is not required to remain constant like the true energy of the many-particle system (in the absence of a time-dependent external field, of course).

How does this happen in our 2D model system? The exact TDKS system has to somehow reproduce the amplitude modulations of the time-dependent dipole moment, but it cannot do so through a simple superposition of oscillations associated with single and double excitations, as it happens in the full two-electron Schrödinger equation: there are no double excitations in the TDKS system. Instead, the TDKS system has to produce the beating pattern in $d(t)$ through the action of the xc potential. In other words, the dynamical part of the xc potential, $V_{\mathrm{xc}}^{\mathrm{dyn}}$, acts in a sense like an "external" potential which alternatingly drives and suppresses the charge-density oscillations of the system in order to increase or diminish the amplitude of $d(t)$. The periodic fluctuations of the adiabatic energy $E_{a}(t)$ serve as an indicator for this behavior.

\section{Memory effects and dissipation}

In the previous subsections, we have seen that the ALDA fails to reproduce some key features of the dynamics of the two-electron system, related to the fact that it misses the double excitations. We have also seen that the exact xc potential makes up for this through nonadiabatic contributions. Let us now see how the nonadiabatic ALDA+M approximation of C-TDDFT performs, which was described in Sec. II B 2.

Figure 7 shows the time-dependent dipole moment for 
the charge-density oscillations of the same 2D quantum strips discussed above but now calculated using the nonadiabatic ALDA+M xc potential [Eq. (31)]. In both cases, the dipole oscillations are exponentially damped, similar to what was previously observed for plasmon oscillations in a semiconductor quantum well. ${ }^{15}$ Representing the dipole moment as $d(t) \sim d_{0} \cos (\omega t) e^{-\Gamma t}$, we find a damping rate of about $\Gamma$ $=0.0007$ for the $L=50$ strip and $\Gamma=0.00055$ for the $L=100$ strip. As expected, the system with $L=100$ has a somewhat weaker damping, due to its lower particle density, which reduces the probability of electron-electron scattering.

The long dashed lines in Fig. 6 show the adiabatic energy $E_{a}(t)$, calculated with ALDA + M. In both quantum strip systems, the energy is dissipated at an exponential rate, $E_{a}(t)=E_{a}(0) e^{-2 \Gamma t}$. As discussed in the previous subsection, the exact adiabiatic energy oscillates, but on average there is no dissipation. These results clearly show that the nonadiabatic ALDA+M functional fails for our finite two-electron system. It results in an unphysical damping of the chargedensity oscillations.

Technically, the dissipation arises from the fact that $V_{\mathrm{xc}}^{M}$ is a velocity-dependent potential. As was discussed in Ref. 6, the history dependence of $V_{\mathrm{xc}}^{M}$, which is governed by the memory kernel $Y\left(n, t-t^{\prime}\right)$, is such that it accounts for both dissipative and elastic properties of the electron liquid. The microscopic properties of the many-body system enter through the frequency-dependent xc kernel of the homogeneous electron gas, $f_{\mathrm{xc}}(\omega)$. This function describes dynamical processes of the homogeneous electron gas that go beyond single-particle excitations, i.e., $f_{\mathrm{xc}}(\omega)$ contains the physics of multiple particle-hole excitations. In principle, this should be a good thing, since we have seen from our model that the main defect of the ALDA is the absence of double excitations. But in spite of this, the ALDA $+M$ does not work in our system. How can one understand this? An answer will emerge from a discussion of the thermodynamic limit in the following subsection.

\section{Thermodynamic limit}

The central reason for the failure of the ALDA+M for finite systems is the fact that it is based on the homogeneous electron gas, i.e., a reference system of infinite extent. Recall that the ALDA+M functional [Eq. (31)] originates from the VK formalism, which expresses the xc vector potential as a functional of the local density and velocity field, using the xc viscosity coefficients of the homogeneous electron liquid. Thus, even though the ALDA+M xc scalar potential is a nonlocal functional of the density [Eq. (32)] and thus sensitive to the size of the system under study, the VK approach implies that exchange and correlation are treated locally as if the system were infinite.

In our case of the 2D quantum strip, the currents flow only along the $z$ direction, and the density is independent of $x$. Thus, $V_{\mathrm{xc}}^{M}(z, t)$ as well as the exact $V_{\mathrm{xc}}(z, t)$ are independent of $x$. However, in contrast to the exact xc potential, $V_{\mathrm{xc}}^{M}(z, t)$ cannot distinguish between a finite and an infinite quantum strip, as long as the density $n(z, t)$ across the strip is the same in both systems.
We can carry out the thought experiment of increasing the length $L$ of the strip and simultaneously adding electrons to keep the same $n(z, t)$ as for the two-electron system. The ALDA and the ALDA+M xc potentials would be unchanged, likewise the Hartree potential, and we would find exactly the same charge-density oscillations across the strip. In particular, the damping in ALDA $+M$ would be the same, irrespective of the length of the strip.

On the other hand, the dynamics of the exact many-body system will change dramatically if we increase both $L$ and the particle number. The time-dependent many-body wave function will contain not only single and double excitations but a vast number of multiple excitations. The density of levels in the excitation spectrum will grow and eventually turn into a continuum. Recall that for the two-electron system, we explained the periodic amplitude modulation of the charge-density oscillation through a superposition of two frequencies associated with the dominant single and double excitations. If the system size grows, many more such transitions will play a role, and we will have to form a coherent superposition of many close-lying oscillators. The resulting beating pattern will become more complex and seem difficult to predict.

However, we can get a clue from comparing the cases of $L=50$ and $L=100$. For the longer strip, the modulation period increases, i.e., the recurrence time becomes longer. In the limit of infinite system size, this suggests that the recurrence time will in fact become infinite. In other words, the charge-density oscillations will be irreversibly damped.

But where does the energy go? Since the purely electronic damping that we consider here can be viewed as a beat with infinite recurrence time, the energy is not lost but remains in the system. In the free charge-density oscillations under study, the wave function can be expressed as a linear superposition of field-free many-body states, each of which carries a time-dependent phase $\exp \left(-i E_{j}^{f} t\right)$ [this is a generalization of Eq. (17) to $N$ particles]. Thus, the energy is, from the very beginning when the oscillation is triggered, shared in a fixed manner among all excited-state configurations that make up the time-dependent many-body wave function. In turn, the charge-density oscillation is a coherent superposition of a continuum of single and multiple particle-hole excitations, which steadily run out of phase. This reduces the amplitude of the collective mode due to destructive interference.

To give a simple illustration, consider the case of an exponential damping of the time-dependent dipole moment, $d(t)=d_{0} \cos (\omega t) e^{-\Gamma t}$. We can carry out a Fourier analysis of the spectral content of $d(t)$, and the result is that $d(t)$ arises from a superposition of a continuum of oscillators whose frequency distribution has a Lorentzian shape of half-width $\Gamma$ centered around $\omega$.

We thus see that the unphysical damping of the ALDA $+\mathrm{M}$ in finite systems derives from the coupling of the collective electron dynamics to continua of multiple particlehole excitations; these continua are implicitly contained in the viscosity coefficients of the homogeneous electron liquid, which are evaluated for the local density at each point. For a physically accurate description of finite systems, where the 
excitation spectrum is discrete, no such coupling to particlehole continua should occur.

On the other hand, the VK formalism of C-TDDFT, per construction, becomes exact in the limit of an extended system whose ground-state density, as well as the inhomogeneity of the time-dependent perturbation, slowly varies in space. ${ }^{7,9,10}$ For such a system, the damping of the plasmon amplitude will be correctly described. In the many-body system, the damping occurs directly through interference within the continuum of multiple excitations, but in C-TDDFT, it has to happen in a more indirect way, since the coupling to the local electron-hole continua is only implicit in the $\mathrm{xc}$ viscosity coefficients. In practice, one finds that the nonadiabatic piece of the xc potential acts like an external damping force. The outcome, i.e., the behavior of the time-dependent density $n(z, t)$, is the same.

\section{CONCLUSION}

We have presented a simple two-electron system which has the appealing feature of being exactly solvable with modest computational cost and rich enough to provide new insight into the problem of dissipation of collective electron dynamics. From the point of view of the exact timedependent many-body wave function, plasmon dissipation occurs through a superposition of a continuum of oscillators, associated with transitions between multiply excited states, which slowly and irreversibly run out of phase. The phenomenon can be viewed like a beat, but with an infinitely long recurrence time. Consequently, there is no loss of energy in the many-body system, and in a sense, not even a redistribution into other degrees of freedom.

From a TDDFT point of view, all we can say is that we have a time-dependent density which produces a timedependent dipole moment whose amplitude steadily decreases. The exact TDKS system accomplishes this through a nonadiabatic xc potential which acts like a damping force. As a result, adiabatic energy of the system is lost, but this is the price we have to pay to reproduce the exact density.

The ALDA $+\mathrm{M}$ xc functional of C-TDDFT has been constructed for infinite systems and becomes exact in the appropriate limits. ${ }^{7}$ For finite systems, it introduces a spurious damping of electron dynamics. For example, if the method is applied to atoms, one obtains excitation energies with finite linewidths. ${ }^{30}$ On the other hand, the static limit of the VK functional ${ }^{7}$ seems to work well for polarizabilities of polymers. ${ }^{13,14}$ Thus, more tests of C-TDDFT are needed to explore its usefulness for practical applications.

However, it seems unlikely that a time-dependent xc functional based on the homogeneous electron gas can correctly describe the subtle aspects of the dynamics of both finite and extended systems that we have discussed in this paper. A more promising approach may be through orbitalbased functionals such as the time-dependent optimized effective potential, ${ }^{31}$ which will be the subject of future studies.

\section{ACKNOWLEDGMENTS}

This work was supported by NSF Grant No. DMR0553485 and by Research Corporation. Discussions with E. K. U. Gross, Robert van Leeuwen, Neepa Maitra, and Giovanni Vignale are gratefully acknowledged.

${ }^{1}$ E. Runge and E. K. U. Gross, Phys. Rev. Lett. 52, 997 (1984).

${ }^{2}$ Time-Dependent Density Functional Theory, Lecture Notes in Physics Vol. 706 edited by M. A. L. Marques, C. A. Ullrich, F. Nogueira, A. Rubio, K. Burke, and E. K. U. Gross (Springer, Berlin, 2006).

${ }^{3}$ F. Furche and K. Burke, in Annual Reports in Computational Chemistry, edited by D. Spellmeyer (Elsevier, Amsterdam, 2005), Vol. 1, p. 19.

${ }^{4}$ N. T. Maitra, F. Zhang, R. J. Cave, and K. Burke, J. Chem. Phys. 120, 5932 (2004).

${ }^{5}$ N. T. Maitra, J. Chem. Phys. 122, 234104 (2005).

${ }^{6}$ C. A. Ullrich and I. V. Tokatly, Phys. Rev. B 73, 235102 (2006).

${ }^{7}$ G. Vignale and W. Kohn, Phys. Rev. Lett. 77, 2037 (1996).

${ }^{8}$ J. F. Dobson, M. J. Bünner, and E. K. U. Gross, Phys. Rev. Lett. 79, 1905 (1997).

${ }^{9}$ G. Vignale, C. A. Ullrich, and S. Conti, Phys. Rev. Lett. 79, 4878 (1997).

${ }^{10}$ C. A. Ullrich and G. Vignale, Phys. Rev. B 65, 245102 (2002); 70, 239903(E) (2004).

${ }^{11}$ Y. Kurzweil and R. Baer, J. Chem. Phys. 121, 8731 (2004).

${ }^{12}$ I. V. Tokatly, Phys. Rev. B 71, 165104 (2005); 71, 165105 (2005).

${ }^{13}$ M. van Faassen, P. L. de Boeij, R. van Leeuwen, J. A. Berger, and J. G. Snijders, Phys. Rev. Lett. 88, 186401 (2002); J. Chem. Phys. 118, 1044 (2003).

${ }^{14}$ M. van Faassen, Int. J. Mod. Phys. B 20, 3419 (2006).

${ }^{15}$ H. O. Wijewardane and C. A. Ullrich, Phys. Rev. Lett. 95, 086401 (2005).

${ }^{16}$ R. D'Agosta and G. Vignale, Phys. Rev. Lett. 96, 016405 (2006).

${ }^{17}$ B. Tanatar and D. M. Ceperley, Phys. Rev. B 39, 5005 (1989).

${ }^{18}$ C. Attaccalite, S. Moroni, P. Gori-Giorgi, and G. B. Bachelet, Phys. Rev. Lett. 88, 256601 (2002).

${ }^{19}$ Z. Qian and G. Vignale, Phys. Rev. B 65, 235121 (2002).

${ }^{20}$ A. Holas and K. S. Singwi, Phys. Rev. B 40, 158 (1989).

${ }^{21}$ E. K. U. Gross and W. Kohn, Phys. Rev. Lett. 55, 2850 (1985); 57, 923(E) (1986).

${ }^{22}$ I. D'Amico and G. Vignale, Phys. Rev. B 59, 7876 (2001).

${ }^{23}$ C. Filippi, C. J. Umrigar, and M. Taut, J. Chem. Phys. 100, 1290 (1994).

${ }^{24}$ In the presence of time-dependent external potentials, the definition of a time-dependent energy is less obvious since the associated many-body Hamiltonian no longer represents the energy operator of the system. See A. Böhm, Quantum Mechanics, 3rd ed. (Springer, New York, 2001).

${ }^{25}$ S. K. Ghosh and A. K. Dhara, Phys. Rev. A 38, 1149 (1988).

${ }^{26}$ G. Vignale, Phys. Rev. B 70, 201102 (2004).

${ }^{27}$ N. T. Maitra, K. Burke, and C. Woodward, Phys. Rev. Lett. 89, 023002 (2002).

${ }^{28}$ P. Hessler, J. Park, and K. Burke, Phys. Rev. Lett. 82, 378 (1999); 83, 5184(E) (1999).

${ }^{29}$ P. Hessler, N. T. Maitra, and K. Burke, J. Chem. Phys. 117, 72 (2002).

${ }^{30}$ C. A. Ullrich and K. Burke, J. Chem. Phys. 121, 28 (2004).

${ }^{31}$ C. A. Ullrich, U. J. Gossmann, and E. K. U. Gross, Phys. Rev. Lett. 74, 872 (1995) 